\title{
STUDY OF SERUM MAGNESIUM LEVELS IN CRITICALLY ILL PATIENTS
}

\author{
Nikhil Elenjickal1, Devpriya Lakra²
}

${ }^{1}$ Resident, Department of Medicine, Pt. JNM Medical College, Raipur.

2Professor, Department of Medicine, Pt. JNM Medical College, Raipur.

\begin{abstract}
\section{BACKGROUND}

Magnesium is the fourth most abundant cation in the body. Hypomagnesaemia is common in critically ill patients, and there is strong, consistent clinical evidence that it is associated with increased need for mechanical ventilation, prolonged ICU stay and increased mortality. There is a current need to expand our knowledge with regards to magnesium homeostasis and its impact on survival and prognosis of critically ill patients.

Aims and Objectives- To study the serum total magnesium in critically ill patients and to assess the primary critical medical conditions associated with abnormalities of serum magnesium and to correlate serum magnesium levels with patient outcome in terms of length of stay and mortality.
\end{abstract}

\section{MATERIALS AND METHODS}

This was an observational study carried out in the medical intensive care unit at a tertiary care hospital. A total of 95 patients were included in the study during a 2-year period. Serum magnesium levels were recorded on the day of admission to intensive care unit. They were followed up to assess associated electrolyte abnormalities, the length of stay and mortality.

\section{RESULTS}

At admission, the incidence of hypomagnesaemia was $52.6 \% ; 27.3 \%$ patients had hypermagnesaemia, while $20 \%$ patients had normomagnesaemia. A significantly higher APACHE II Score was recorded in subjects with hypomagnesaemia compared to those with normomagnesaemia. Hyponatraemia was seen in $76 \%$ patients, while $29.4 \%$ patients had hypocalcaemia and $44.2 \%$ had hypokalaemia. Length of ICU stay was found to be significantly higher in subjects with hypomagnesaemia compared to those with normomagnesaemia and hypermagnesaemia $(\mathrm{p}<0.0001)$. Mortality was higher in patients with hypomagnesaemia $(80 \%)$, while that in the hypermagnesaemia group had $53.8 \%$ mortality.

\section{CONCLUSION}

This study revealed the prevalence of dysmagnesaemia in the critically ill population of ICU and highlighted the role of magnesium monitoring. This study also showed that hypomagnesaemia was associated with higher APACHE II score, longer ICU stay and greater mortality.

\section{KEYWORDS}

Magnesium; Hypomagnesaemia; Critical Illness; Mortality; Dysmagnesaemia.

HOW TO CITE THIS ARTICLE: Elenjickal N, Lakra D. Study of serum magnesium levels in critically ill patients. J. Evolution Med. Dent. Sci. 2017;6(42):3332-3336, DOI: 10.14260/Jemds/2017/722

\section{BACKGROUND}

Magnesium is the fourth most abundant cation in the body and the second most abundant intracellular cation after potassium. ${ }^{1}$ It is an element that is constantly being "rediscovered." 1 It is an essential element for the utilisation of energy in the organic world.

Aerobic organisms then use the oxygen to release the energy stored in organic nutrients (including carbohydrates) and this energy is stored as Adenosine Triphosphate (ATP). The release of energy from ATP requires magnesium, which is an essential cofactor for the ATPase enzymes that hydrolyse ATP. Therefore, magnesium is essential for providing us with energy and for allowing us to utilise

Financial or Other, Competing Interest: None.

Submission 19-04-2017, Peer Review 14-05-2017,

Acceptance 19-05-2017, Published 25-05-2017.

Corresponding Author:

Dr. Nikhil Elenjickal,

Intern Hostel, Room 16,

Pt. JNM Medical College Campus,

Jail Road, Raipur-492001.

E-mail: drnikhiljohn@gmail.com

DOI: $10.14260 /$ jemds $/ 2017 / 722$ this energy to sustain life. The total body magnesium level of an average adult is $24 \mathrm{~g}$ or $1000 \mathrm{mmol} .^{2}$ Approximately, $60 \%$ of the body's magnesium is present in bone, $20 \%$ is in muscle and another $20 \%$ is in soft tissue. ${ }^{2}$

Magnesium depletion is described as "the most underdiagnosed electrolyte abnormality" in current medical practice, yet it is frequently overlooked and undercorrected. Magnesium, hence, has been given various terms such as"The disregarded cation."3 and "The forgotten electrolyte."4 Hypomagnesaemia is common in critically ill patients, and there is strong, consistent clinical evidence, largely from observational studies showing that hypomagnesaemia is significantly associated with increased need for prolonged ICU stay and increased mortality.

Serum magnesium monitoring may have prognostic and perhaps therapeutic implications, because critically ill patients are predisposed to both symptomatic and asymptomatic magnesium deficiency that can lead to some important clinical consequences (such as hypokalaemia, cardiac arrhythmias, hypocalcaemia, neurotoxicity and psychiatric problems), ultimately increasing the morbidity and mortality. 
There is lack of data in the Indian literature addressing this common electrolyte abnormality. With this in mind, we conducted an observational study in our institute to find the prevalence of dysmagnesaemia in the critically ill population, other electrolyte imbalances and association with length of ICU stay and mortality.

\section{Aims and Objectives}

- To study the serum total magnesium in critically ill patients.

- To assess the primary critical medical conditions associated with abnormalities of serum magnesium.

- To correlate serum magnesium level with patient outcome in terms of- Length of stay in MICU/ICCU and Mortality.

- To detect other electrolyte abnormalities (e.g. hypocalcaemia, hypokalaemia) associated with dysmagnesaemia.

\section{MATERIALS AND METHODS}

This observational study was conducted in the Department of Medicine, Dr. B.R.A.M. Hospital, Raipur among the critically ill patients admitted in MICU and ICCU from 2014 - 2016. Ethical clearance was obtained and the study was initiated. Patients were selected based on the inclusion criteria. Written informed consent was taken from all the patients. A complete history, physical examination and systemic examination was done in all the patients. Amongst patients who fulfilled the inclusion criteria, $5 \mathrm{~mL}$ of venous blood was taken at the time of hospital admission for blood investigations and other routine examinations. Patient's baseline information was recorded at admission including age, sex, blood pressure (in $\mathrm{mmHg}$ ), and random glucose level at admission (in $\mathrm{mg} / \mathrm{dL}$ ). The blood sample collected was used for investigations like complete blood count, serum electrolytes, serum calcium, renal function tests, liver function tests and serum magnesium. APACHE II score was calculated on the day of admission to MICU/ICCU. Arterial blood sample of $2 \mathrm{~mL}$ was collected in all patients for $\mathrm{ABG}$ analysis. Duration of stay in the ICU was recorded. The study did not interfere with the patient management in the ICU.

\section{Statistical Analysis}

SPSS Software for Windows ${ }^{\mathrm{TM}}$ Ver. 17, IBM $^{\mathrm{TM}}$ Corp NY and Microsoft Excel $^{\mathrm{TM}}$ 2007, Microsoft ${ }^{\circledR}$ Inc. USA was used to perform the statistical analysis.

Analysis was done by using description and inferential statistics. All the data were expressed in mean \pm standard deviation (SD). The analysis was performed by using Student's t-test for the difference of means, Chi-square test and correlation. ANOVA followed by Tukey's HSD test was used to test the significance of difference between more than two parameters in parametric data.

\section{Methodology}

At alkaline $\mathrm{pH}$, magnesium reacts with Xylidyl blue to form a red coloured compound. This method developed originally by Mann and Yoe, 5 is based upon the formation of a water soluble red-purple chelate of magnesium and Xylidyl blue. The intensity of the developed colour is proportional to the serum magnesium concentration. Sample was mixed with the reagent and incubated at $25^{\circ} \mathrm{C}$ for 5 minutes. The coloured complex formed by magnesium with the monoreagent was read on a colorimeter in $1 \mathrm{~mL}$ cuvettes. The absorbance of the sample (AT) and Standard (AS) was measured against Blank at $505 \mathrm{~nm}$. The results were expressed in $\mathrm{mg} / \mathrm{dL}$. The reference range of serum magnesium for this study was between $1.7-2.4 \mathrm{mg} / \mathrm{dL}$. Serum magnesium less than 1.7 was considered to be hypomagnesaemia and more than 2.4 was considered to be hypermagnesaemia.

\section{RESULTS}

This prospective study included 95 patients admitted in the intensive care unit. Out of these 95 subjects, 30.5\% patients were in the age group $</=30 \mathrm{yrs}$., $15.8 \%$ in the age group of $41-50$ and $6.3 \%$ in the age group of $>70$ yrs. Figure 1 shows that majority of the study population suffered from cerebrovascular accidents (18.8\%) (including infarct and haemorrhage) followed by COPD with acute exacerbation $(12.5 \%)$. The least common diagnoses included Diabetes with Diabetic Ketoacidosis (1\%) and hanging with Hypoxic Encephalopathy (1\%) with 1 patient each in both.

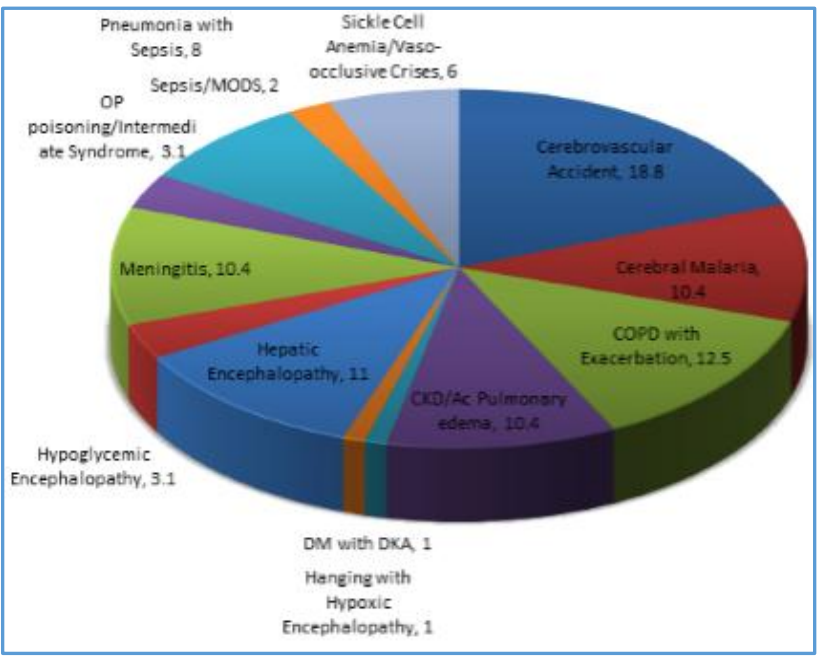

Figure 1. Diagnoses of Patients admitted in the Intensive Care Unit

Out of the total 95 subjects $52.63 \%$ patients had hypomagnesaemia, $27.37 \%$ had hypermagnesaemia and $20 \%$ had normomagnesaemia. Average serum magnesium recorded was $1.95 \mathrm{mg} / \mathrm{dL}$. In our study, 73 patients had hyponatraemia ( $\mathrm{Na}<135 \mathrm{mmol} / \mathrm{L})$ and 22 patients had normal sodium levels. No significant difference was recorded in the three groups, viz. Hypomagnesaemia, Normomagnesaemia and Hypermagnesaemia. 42 patients of the study population had hypokalaemia $(\mathrm{K}+<3.5 \mathrm{mmol} / \mathrm{L})$, while 50 patients had normal potassium and 3 had hyperkalaemia ( $\mathrm{K}+>5.5 \mathrm{mmol} / \mathrm{L})$. No significant difference was recorded in the three groups, viz. Hypomagnesaemia, Normomagnesaemia and Hypermagnesaemia. 43 patients (45.2\%) had hypocalcaemia and 52 patients (54.7\%) had normal serum calcium levels. Mean serum calcium was 8.76 $\mathrm{mg} / \mathrm{dL}$. Lowest serum calcium recorded was $8 \mathrm{mg} / \mathrm{dL} .28$ patients with Hypomagnesaemia had Hypocalcaemia as well. 


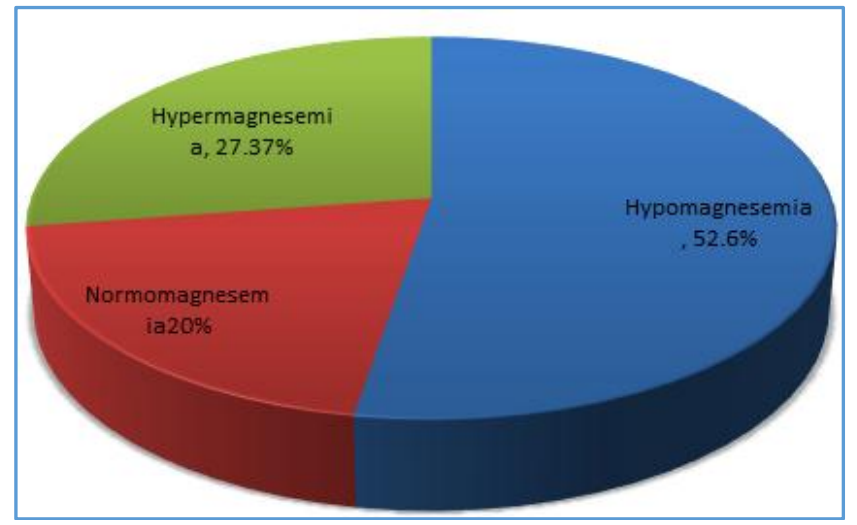

Figure 2. Serum Magnesium Levels in Patients

A significantly higher APACHE II Score was recorded in subjects with hypomagnesaemia compared to those with normomagnesaemia $(\mathrm{p}<0.0001)$. Length of ICU stay was an important outcome variable that was chosen for this study as it depends on multiple factors including electrolyte homeostasis. Length of ICU stay was found to be significantly higher in subjects with hypomagnesaemia compared to those with normomagnesaemia $(\mathrm{p}<0.0001)$ and hypermagnesaemia $(\mathrm{p}<0.0001)$.

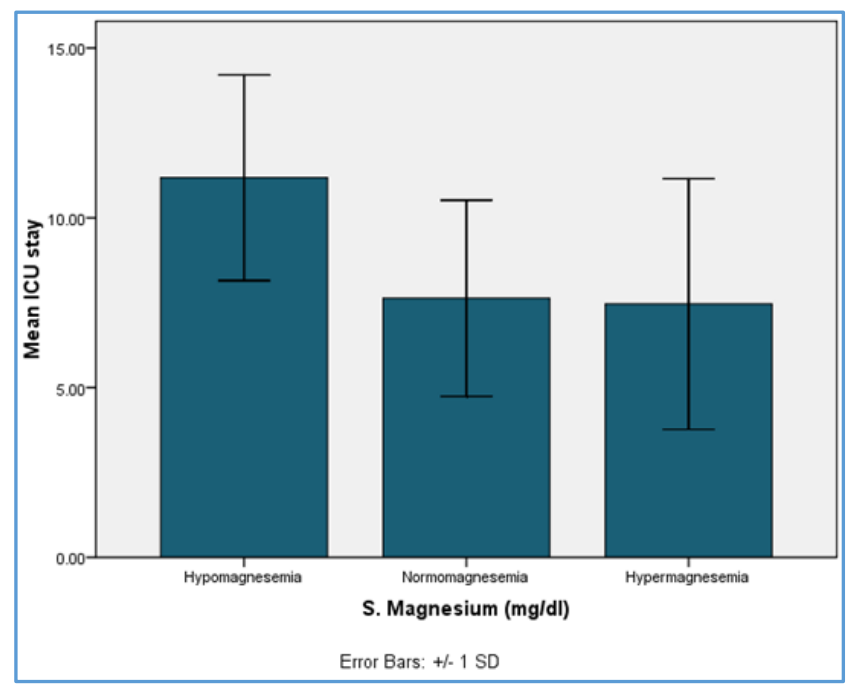

Figure 3. ICU Stay and association with Serum Magnesium Levels

Higher frequency of mortality in subjects was found to exist $(p=0.002)$ with hypomagnesaemia and hypermagnesaemia. 34 patients (35.7\%) survived, while 61 patients (64.21\%) succumbed to their illnesses. Amongst the hypomagnesaemia group mortality reached to an astounding $80 \%$, while that in the hypermagnesaemia group had $53.8 \%$ mortality. $36.8 \%$ patients with normomagnesaemia succumbed to their illnesses.

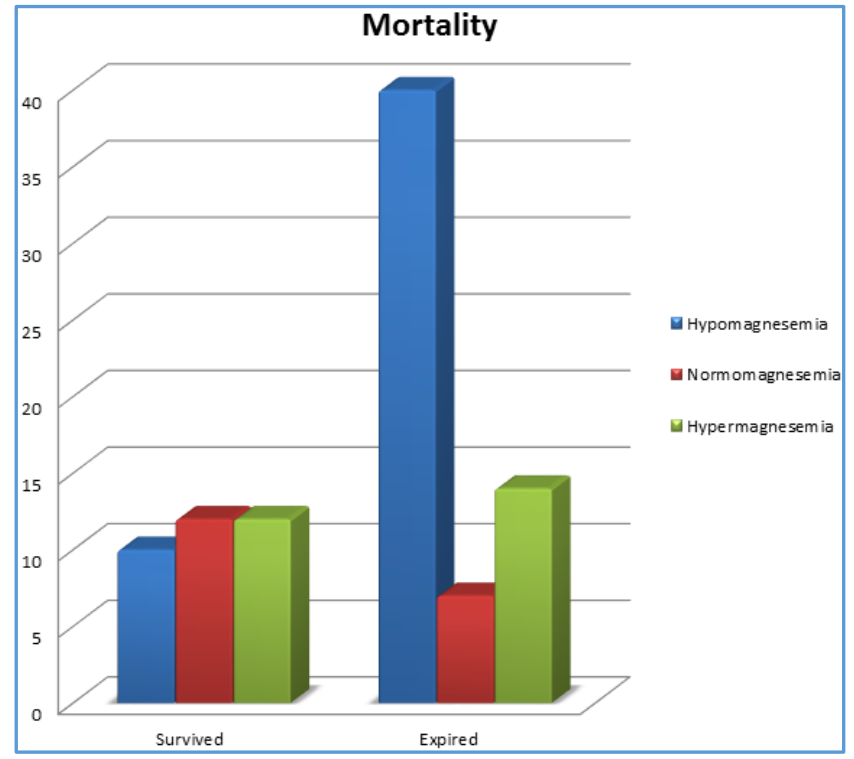

Figure 4. Association of Mortality with Serum Magnesium Levels

\section{DISCUSSION}

Magnesium is the fourth most common cation in the body and the second most common intracellular cation after potassium. It has a fundamental role as a co-factor in more than 300 enzymatic reactions involving energy metabolism and nucleic acid synthesis. ${ }^{6}$ It is also involved in several processes including hormone receptor binding; gating of calcium channels; transmembrane ion flux and regulation of adenylate cyclase; muscle contraction; neuronal activity; control of vasomotor tone; cardiac excitability; and neurotransmitter release.

The significance of magnesium and its relationship to the origin of life has been traced to the formation of chlorophyll with magnesium at the centre of the molecule, and its interaction with Adenosine Triphosphate (ATP). Aerobic organisms then use the oxygen to release the energy stored in organic nutrients (including carbohydrates), and this energy is stored as Adenosine Triphosphate (ATP). The release of energy from ATP requires magnesium, which is an essential cofactor for the ATPase enzymes that hydrolyse ATP. Therefore, magnesium is essential for providing us with energy and for allowing us to utilise this energy to sustain life. It also maintains neuromuscular excitability and it is important for maintenance of cardiac function. By regulating enzymes controlling intracellular calcium, Mg affects smooth muscle vasoconstriction.

In this study, the prevalence of Hypomagnesaemia was found to be $52.6 \%$ (50 subjects). $27.37 \%$ (26 subjects) had hypermagnesaemia, while $20 \%$ (19) of the subjects were normomagnesaemic. An observational study on 102 medical ICU patients by Reinhart et $\mathrm{al}^{7}$ showed that hypomagnesaemia was present in $20 \%$ of patients, while hypermagnesaemia was present in $9 \%$ of patients, and of all ions $\mathrm{Mg}$ had the highest prevalence of abnormal values. Another prospective observational study on 100 ICU patients by Limaye et $\mathrm{al}^{8}$ showed that on ICU admission, $52 \%$ of patients had hypomagnesaemia, $41 \%$ had normal serum $\mathrm{Mg}$ levels and $7 \%$ had hypermagnesaemia. Chernow et $\mathrm{al}^{9}$ measured serum Mg levels in blood samples from 193 ICU 
patients and found that 117 of 193 (61\%) had hypomagnesaemia on ICU admission.

Subhraprakashpramanik et $\mathrm{al}^{10}$ in a study of 100 critically ill patients found the prevalence of hypomagnesaemia as $53 \%$. The mean level of serum magnesium was $1.23 \pm 0.18$ $\mathrm{mg} / \mathrm{dL}$ in hypomagnesaemic patients compared to $1.89 \pm 0.12$ $\mathrm{mg} / \mathrm{dL}$ in normomagnesaemic group of patients.

A significantly higher APACHE II Score was found using Tukey's HSD test in subjects with hypomagnesaemia compared to those with normomagnesaemia ( $p<0.0001$ ). The mean APACHE II score was 22.93. Highest APACHE II score recorded was 27, while the lowest score was 19 . A similar result was obtained by Sunil Kumar et al ${ }^{11}$ in their study with the mean APACHE II score between hypomagnesaemic and normomagnesaemic group was 21.82 \pm 5.90 and $19.26 \pm 4.37$. Safavi $M$ et al ${ }^{12}$ showed in their study that Hypomagnesaemic patients had more severe organ dysfunction and higher APACHE II score than the other patients. This may be explained by a strong association of hypomagnesaemia with sepsis and septic shock, common cause of death in the ICU patient. This may be explained by a strong association of hypomagnesaemia with sepsis and septic shock, a common cause of death in the ICU patients.

In this study, comparison of length of ICU stay with magnesium levels was performed using ANOVA. Significant difference was noted between three groups regarding ICU stay $(p<0.0001)$. Further post-hoc analysis was performed using Tukey's HSD test and ICU stay was found to be significantly higher in subjects with hypomagnesaemia compared to those with normo ( $p<0.0001)$ and hypermagnesaemia $(\mathrm{p}<0.0001)$. The mean ICU stay was 9.45 days with the shortest stay being 2 days and the longest stay being 16 days. Patients with hypomagnesaemia had a significantly longer duration of ICU stay as compared to that of those with normomagnesaemia. A similar result was obtained by Safavi M et al,12 who showed in their study that there was significant difference in ICU and hospital length of stay or mortality among the hypomagnesaemic and normomagnesaemic groups. The average ICU stay amongst patients with low magnesium was $9.16 \pm 0.53$ days, while that in patients with normal magnesium was $5.71 \pm 0.55$ days. Subhraprakashpramanik et al ${ }^{10}$ revealed in their study that the length of ICU stay and total hospital duration both were longer in hypomagnesaemic patients. In the study by Sunil Kumar et al,11 the investigators noted a significant difference in the length of ICU stay in hypomagnesaemic and normomagnesaemic group of patients $(5.46 \pm 5.75$ days vs. $3.93 \pm 3.88$ days, $\mathrm{P}=0.0002$ ) .

Mortality was another important outcome variable that was chosen for this study. In this study, association of mortality with serum magnesium levels was analysed using chi-square test. Significant association was found to exist $(p=0.002)$ indicating a higher frequency of mortality in subjects with hypomagnesaemia and hypermagnesaemia. In this study, out of 95 patients 34 patients (35.7\%) survived, while 61 patients (64.21\%) succumbed to their illnesses. Amongst the hypomagnesaemia group, mortality reached to an astounding $80 \%$, while that in the hypermagnesaemia group had $53.8 \%$ mortality; $36.8 \%$ patients with normomagnesaemia succumbed to their illnesses. This result clearly shows that dysmagnesaemia is an important factor involved in the mortality of the patients.

The study by Seyed Ali Javed Mousavi et al ${ }^{13}$ concluded that the mortality rate was higher in patients who had abnormal magnesium levels. The mortality rate was higher in patients who had lower magnesium levels (Mann-Whitney; $\mathrm{p}=0.000$ ). Mir Sadaqat Hassan Zafar et $\mathrm{al}^{14}$ showed that the mortality in the hypomagnesaemic group was $76.47 \%$, while that of normomagnesaemic group was $36 \%(\mathrm{P}=0.004)$. Occurrence of magnesium deficiency in critical illness correlates with higher morbidity and mortality. Higher mortality rate was detected in hypomagnesaemia patients when compared with normomagnesaemic patients by Limaye et $\mathrm{al}^{8}(57 \%$ vs. $31 \%)$ as well.

\section{Limitations of this Study}

Serum magnesium levels were recorded only on the day of admission to intensive care unit. Followup magnesium levels were not done. Secondly, this was a descriptive noninterventional study. A large multicentric, randomised, double-blind, interventional, trial for magnesium supplementation in critically ill patients with hypomagnesaemia is needed in future to evolve consensus/guidelines for treatment of hypomagnesaemia in critically ill.

\section{CONCLUSION}

This study revealed the prevalence of dysmagnesaemia in the critically ill population of ICU. This study highlights the role of magnesium monitoring in critical illness. It adds to the scarce Indian data with regards to the magnesium homeostasis in ICUs. This study also showed that hypomagnesaemia was associated with higher APACHE II score and longer ICU stay. A significant proportion of the mortality was seen in both hypomagnesaemic group and hypermagnesaemic group. Hypomagnesaemia should be identified and corrected, because it is associated with increased adverse events and high mortality in critically ill patients. Physicians should maintain a high index of suspicion for hypomagnesaemia and the need for Mg replacement therapy.

\section{ACKNOWLEDGEMENT}

The authors would like to thank patients for their participation in the study. Authors also acknowledge the immense help received from the scholars whose articles are cited and included in references of this manuscript.

\section{REFERENCES}

[1] Charles BS, Menon I, Girish TS, et al. Hypomagnesemia in the ICU-does correction matter? J Assoc Physicians India 2016;64(11):15-19.

[2] Wallach S. Availability of body magnesium during magnesium deficiency. Magnesium 1988;7(5-6): 262-70.

[3] Walsh SB, Zdebik AA, Unwin RJ, et al. Magnesium: the disregarded cation. Mayo Clin Proc 2015;90(8):993-5.

[4] Geiderman JM, Goodman SL, Cohen DB. Magnesiumthe forgotten electrolyte. JACEP 1979;8(5):204-8. 
[5] Mann CK, Yoe JH. Spectrophotometric determination of magnesium with sodium 1-Azo-2-hydroxy-3-(2, 4dimethylcarboxanilido)-naphthalene-1- (2-hydroxyl benzene- 5-sulfonate). Analytical Chem 1956; 28(2): 202-5.

[6] Laurant P, Touyz RM. Physiological and pathophysiological role of magnesium in the cardiovascular system: implications in hypertension. J Hypertens 2000;18(9):1177-91.

[7] Reinhart RA, Desbiens NA. Hypomagnesemia in patients entering the ICU. Crit Care Med 1985; 13(6): 506-7.

[8] Limaye CS, Londhey VA, Nadkart MY, et al. Hypomagnesemia in critically ill medical patients. J Assoc Physicians India 2011;59:19-22.

[9] Chernow B, Bamberger S, Stoiko $M$, et al. Hypomagnesemia in patients in postoperative intensive care. Chest 1989;95(2):391-7.
[10] Subhraprakashpramanik, Dey AK, Pijushkantimandal, et al. Prevalence of hypomagnesemia and its predictive prognostic value in critically Ill medical patients. IOSR Journal of Pharmacy 2014;4(1):01-05.

[11] Kumar S, Honmode A, Jain S, et al. Does magnesium matter in patients of medical intensive care unit: a study in rural central India. Indian J Crit Care Med 2015;19(7):379-83.

[12] Safavi M, Honarmand A. Admission hypomagnesemiaimpact on mortality and morbidity in critically ill patients. Middle East J Anaesthesiol 2007;19(3): 645-60.

[13] Mousavi SAJ, Salimi S, Rezai M. Serum magnesium level impact on the outcome of patients admitted to the intensive care unit. Tanaffos 2010;9(4):28-33.

[14] Zafar MSH, Wani JI, Karim R, et al. Significance of serum magnesium levels in critically ill patients. Int J Appl Basic Med Res 2014;4(1):34-7. 